PROCEEDINGS OF THE

AMERICAN MATHEMATICAL SOCIETY

Volume 139, Number 1, January 2011, Pages 51-62

S 0002-9939(2010)10619-6

Article electronically published on August 23, 2010

\title{
TAUTOLOGICAL PAIRINGS ON MODULI SPACES OF CURVES
}

\author{
RENZO CAVALIERI AND STEPHANIE YANG
}

(Communicated by Ted Chinburg)

\begin{abstract}
We discuss analogs of Faber's conjecture for two nested sequences of partial compactifications of the moduli space of smooth pointed curves. We show that their tautological rings are one-dimensional in top degree but sometimes do not satisfy Poincaré duality.
\end{abstract}

The structure of the tautological ring of the moduli space of smooth curves of genus $g$ is predicted by the Faber conjecture, which states that $R^{*}\left(\mathcal{M}_{g}\right)$ is Gorenstein with socle in dimension $g-2([\mathrm{Fa}])$. We break this statement into two parts:

Socle: The tautological ring vanishes in high degree and is one-dimensional in top degree:

$$
R^{k}\left(\mathcal{M}_{g}\right)= \begin{cases}0, & \text { if } k>g-2, \\ \mathbb{Q}, & \text { if } k=g-2 .\end{cases}
$$

Poincaré duality: For $0 \leq k \leq g-2$, the bilinear pairing

$$
R^{k}\left(\mathcal{M}_{g}\right) \times R^{g-2-k}\left(\mathcal{M}_{g}\right) \rightarrow R^{g-2}\left(\mathcal{M}_{g}\right)
$$

is nondegenerate.

In FP2, Faber and Pandharipande speculate that the tautological rings of $\overline{\mathcal{M}}_{g, n}$, $\mathcal{M}_{g, n}^{\mathrm{ct}}$ and $\mathcal{M}_{g, n}^{\mathrm{rt}}$ satisfy analogous properties. (Here, $\mathcal{M}_{g, n}^{\mathrm{ct}}$ denotes the moduli space of curves of compact type and $\mathcal{M}_{g, n}^{\mathrm{rt}}$ the moduli space of curves with rational tails.) While the socle statements of these speculations have been proven, the Poincaré duality property remains open. We give evidence that the two properties are not necessarily immediately correlated.

We define two chains of partial compactifications (Definitions 1.2 and 1.3)

$$
\begin{gathered}
\mathcal{M}_{g, n}^{\mathrm{ct}}=\mathcal{M}_{g, n}^{\lambda_{g}} \subseteq \mathcal{M}_{g, n}^{\lambda_{g-1}} \subseteq \cdots \subseteq \mathcal{M}_{g, n}^{\lambda_{1}} \subseteq \overline{\mathcal{M}}_{g, n}, \\
\mathcal{M}_{g, n}^{\mathrm{rt}}=\mathcal{M}_{g, n}^{\mathrm{ch}_{2 g-1}} \subseteq \mathcal{M}_{g, n}^{\mathrm{ch}_{2 g-3}} \subseteq \cdots \subseteq \mathcal{M}_{g, n}^{\mathrm{ch}_{1}} \subseteq \overline{\mathcal{M}}_{g, n}
\end{gathered}
$$

and define their tautological rings by restriction. The main results of this paper address the analog of Faber's conjecture for these spaces. The socle statement extends but Poincaré duality fails for the first cases $\mathcal{M}_{g, n}^{\lambda_{1}}=\mathcal{M}_{g, n}^{\mathrm{ch}_{1}}$ if $g \geq 2$ and $(g, n) \neq 0$.

Proposition 1. For $i=1,2, \ldots, g$,

(1) $R^{k}\left(\mathcal{M}_{g, n}^{\lambda_{i}}\right)=0$ for $k>3 g-3+n-i$ and $R^{3 g-3+n-i}\left(\mathcal{M}_{g, n}^{\lambda_{i}}\right) \cong \mathbb{Q}$,

Received by the editors February 24, 2009 and, in revised form, April 26, 2009; September 1, 2009; December 1, 2009; and January 29, 2010.

2010 Mathematics Subject Classification. Primary 14N35.

(C) 2010 American Mathematical Society Reverts to public domain 28 years from publication 
(2) $R^{k}\left(\mathcal{M}_{g, n}^{\mathrm{ch}_{2 i-1}}\right)=0$ for $k>3 g-2+n-2 i$ and $R^{3 g-2+n-2 i}\left(\mathcal{M}_{g, n}^{\mathrm{ch}_{2 i-1}}\right) \cong \mathbb{Q}$.

Proposition 2. For $g \geq 2$ and $(g, n) \neq(2,0)$, the pairing

$$
R^{g}\left(\mathcal{M}_{g, n}^{\lambda_{1}}\right) \times R^{2 g-4+n}\left(\mathcal{M}_{g, n}^{\lambda_{1}}\right) \rightarrow R^{3 g-4+n}\left(\mathcal{M}_{g, n}^{\lambda_{1}}\right)
$$

is not perfect.

The tautological rings of the other intermediate spaces $\mathcal{M}_{g, n}^{\lambda_{i}}$ are addressed with the following asymptotic result.

Proposition 3. For any fixed $g$ and $2 \leq i \leq g-1$ and for every $n \gg 0$, either the tautological restriction sequence

$$
R^{i-1}\left(\overline{\mathcal{M}}_{g, n} \backslash \mathcal{M}_{g, n}^{\lambda_{i}}\right) \longrightarrow R^{g}\left(\overline{\mathcal{M}}_{g, n}\right) \longrightarrow R^{g}\left(\mathcal{M}_{g, n}^{\lambda_{i}}\right) \longrightarrow 0
$$

is not exact in the middle or the pairing

$$
R^{g}\left(\mathcal{M}_{g, n}^{\lambda_{i}}\right) \times R^{2 g-3-i+n}\left(\mathcal{M}_{g, n}^{\lambda_{i}}\right) \rightarrow R^{3 g-3-i+n}\left(\mathcal{M}_{g, n}^{\lambda_{i}}\right)
$$

is not perfect.

The Chow ring is known to be tautological in codimensions 0 and 1 (M). Therefore, the restriction sequence (6) is exact when $i=1,2$ and Proposition 3 immediately implies:

Corollary 4. If $g \geq 3$ and $i=2$, then the pairing (7) is not perfect for every $n \gg 0$.

The restriction sequence (6) is known to be exact for $\mathcal{M}_{g}$ in degrees $\geq g-1$, and exactness is conjectured in all degrees for the $\mathcal{M}_{g, n}^{\mathrm{rt}}$ and $\mathcal{M}_{g, n}^{\mathrm{ct}}$ ([FP3]). Therefore we expect the following:

Conjecture. For $2 \leq i \leq g-1$, and $(g, n) \neq(2,0)$, the pairing on the tautological rings of $\mathcal{M}_{g, n}^{\lambda_{i}}$ is never perfect.

Remark 1. When $i=1$, we have $\mathcal{M}_{g, n}^{\lambda_{1}}=\mathcal{M}_{g, n}^{\mathrm{ch}_{1}}$. However, the proof of Proposition 3 does not extend in a natural way to $\mathcal{M}_{g, n}^{\mathrm{ch}_{i}}$, for $i>1$. In these cases, Poincaré duality remains an open question.

With Lemma 1.4 we show that for the extremal case $i=g, \mathcal{M}_{g, n}^{\lambda_{g}}=\mathcal{M}_{g, n}^{\mathrm{ct}}$ and $\mathcal{M}_{g, n}^{\mathrm{ch}_{2 g-1}}=\mathcal{M}_{g, n}^{\mathrm{rt}}$. Our results and conjecture therefore address generalizations of spaces that appeared in FP2. The socle statements of the Faber conjectures for $\mathcal{M}_{g, n}^{\mathrm{rt}}, \mathcal{M}_{g, n}^{\mathrm{ct}}$, and $\overline{\mathcal{M}}_{g, n}$ were proved by Graber-Vakil (GV2, $\left.\left.\S 5.5-\S 5.7\right]\right)$ and FaberPandharipande ([FP3 $\S 4.1])$. Prior to these results, the socle statement for $\mathcal{M}_{g}$ was shown by Looijenga and Faber ( $[\mathrm{L} ; \mathrm{Fa}$, Theorem 2$])$. The Poincaré duality property is only known for $g=0$ by Keel ( $[\mathrm{Ke}]$ ), and for $\mathcal{M}_{g}$ for $g \leq 23$ by Faber ([Fa $]$ ).

Remark 2. We also show that for $g \geq 2$, the dimension of the kernel of the map

$$
R^{g}\left(\mathcal{M}_{g, n}^{\lambda_{1}}\right) \rightarrow \operatorname{hom}\left(R^{2 g-4+n}\left(\mathcal{M}_{g, n}^{\lambda_{1}}\right), R^{3 g-4+n}\left(\mathcal{M}_{g, n}^{\lambda_{1}}\right)\right)
$$

becomes arbitrarily large as either $g$ or $n$ grows (Corollary 5). It is easy to see that Poincaré duality fails in arbitrary degrees by taking nonzero elements of high degree in the ideal generated by the kernel of this map. 
Remark 3. Note that $R^{*}\left(\mathcal{M}_{2,0}^{\lambda_{1}}\right)$ is Gorenstein. It is straightforward to check that the intersection matrix for the two generators of $R^{1}\left(\mathcal{M}_{2,0}^{\lambda_{1}}\right)$ is nondegenerate. Since $\pi_{1}^{*}\left(\mathcal{M}_{2,0}^{\lambda_{1}}\right)=\mathcal{M}_{2,1}^{\lambda_{1}}$, Proposition 2 shows that if $\mathcal{M}$ is a moduli space which satisfies Faber's conjecture, its universal family does not necessarily satisfy Faber's conjecture.

Remark 4. The classes $\lambda_{i}$ and $\operatorname{ch}_{2 i-1}$ vanish respectively on $\overline{\mathcal{M}}_{g, n} \backslash \mathcal{M}_{g, n}^{\lambda_{i}}$ and $\overline{\mathcal{M}}_{g, n} \backslash \mathcal{M}_{g, n}^{\mathrm{ch}_{2 i-1}}$. This motivates our notation.

This paper is organized as follows: in the first section we recall some basic definitions and provide references to the existing literature. In each of the subsequent sections we prove the three propositions.

The authors thank Carel Faber and Ravi Vakil for helpful discussions. The research was partially supported by the National Science Foundation, the Gustafsson Foundation, and the Wallenberg Foundation.

\section{BACKGROUND}

The tautological ring $R^{*}\left(\overline{\mathcal{M}}_{g, n}\right)$ is a natural subring of the Chow ring $A^{*}\left(\overline{\mathcal{M}}_{g, n}\right)$ elegantly defined in [FP3]: as $g$ and $n$ vary, the tautological rings form the smallest system of $\mathbb{Q}$-subalgebras of $A^{*}\left(\overline{\mathcal{M}}_{g, n}\right)$ that are closed under pushforward and pullback by the natural forgetful morphisms

$$
\pi_{i}: \overline{\mathcal{M}}_{g, n+1} \rightarrow \overline{\mathcal{M}}_{g, n}
$$

and the gluing morphisms

$$
\begin{gathered}
\iota_{\mathrm{irr}}: \overline{\mathcal{M}}_{g-1, n+2} \rightarrow \overline{\mathcal{M}}_{g, n}, \\
\iota_{g_{1}, n_{1}}: \overline{\mathcal{M}}_{g_{1}, n_{1}+1} \times \overline{\mathcal{M}}_{g_{2}, n_{2}+1} \rightarrow \overline{\mathcal{M}}_{g_{1}+g_{2}, n_{1}+n_{2}} .
\end{gathered}
$$

The tautological ring contains boundary strata $\delta_{\Gamma}$ (fundamental classes of the closure of the locus of curves whose dual graph is $\Gamma$ ), cotangent line classes $\psi_{i}$, Mumford-Morita-Miller $\kappa$ classes, and Chern classes $\lambda_{i}$ of the Hodge bundle. Much is known about the intersection theory of such classes. An excellent, albeit unfinished and unpublished, reference is $[\mathrm{Ko}]$. Other references include $[\mathrm{Fu}], \mathrm{HKK}^{+}$, and $[\mathrm{M}]$.

The following formula will be used in the proof of Proposition 2 .

Lemma 1.1. For any value of $n$ for which the integrals are defined:

$$
\begin{aligned}
\int_{\overline{\mathcal{M}}_{0, n}} \kappa_{n-3} & =1, \\
\int_{\overline{\mathcal{M}}_{1, n}} \kappa_{n} & =\frac{1}{24}, \\
\int_{\overline{\mathcal{M}}_{0, n}} \kappa_{i} \kappa_{n-i-3} & =\left(\begin{array}{c}
n-1 \\
i+1
\end{array}\right)-1, \\
\int_{\overline{\mathcal{M}}_{1, n}} \kappa_{1} \kappa_{n-1} & =\left(\begin{array}{c}
n+1 \\
2
\end{array}\right) \frac{1}{24} .
\end{aligned}
$$

Proof. Equations (12) and (13) follow immediately from the pull-back formula for $\kappa$ classes ([AC, Eq. (1.10)]),

$$
\kappa_{a}=\pi_{n+1}^{*}\left(\kappa_{a}\right)+\psi_{n+1}^{a} .
$$


From this it follows easily that

$$
\begin{aligned}
\int_{\overline{\mathcal{M}}_{0, n}} \kappa_{i} \kappa_{n-i-3} & =\int_{\overline{\mathcal{M}}_{0, n}}\left(\pi_{n+1 *} \psi_{n+1}^{i+1}\right) \kappa_{n-i-3} \\
& =\int_{\overline{\mathcal{M}}_{0, n+1}} \psi_{n+1}^{i+1}\left(\kappa_{n-i-3}-\psi_{n+1}^{n-i-3}\right) \\
& =\int_{\overline{\mathcal{M}}_{0, n+1}} \psi_{n+1}^{i+1}\left(\pi_{n+2 *} \psi_{n+2}^{n-i-2}\right)-\int_{\overline{\mathcal{M}}_{0, n+1}} \psi_{n+1}^{n-2} \\
& =\int_{\overline{\mathcal{M}}_{0, n+2}}\left(\psi_{n+1}-D_{n+1, n+2}\right)^{i+1} \psi_{n+2}^{n-i-2}-\int_{\overline{\mathcal{M}}_{0, n+1}} \psi_{n+1}^{n-2} \\
& =\int_{\overline{\mathcal{M}}_{0, n+2}} \psi_{n+1}^{i+1} \psi_{n+2}^{n-i-2}-0-\int_{\overline{\mathcal{M}}_{0, n+1}} \psi_{n+1}^{n-2} \\
& =\left(\begin{array}{c}
n-1 \\
i+1
\end{array}\right)-1 .
\end{aligned}
$$

The proof for (15) is analogous.

Definition 1.2. $\mathcal{M}_{g, n}^{\lambda_{i}} \subseteq \overline{\mathcal{M}}_{g, n}$ is the locus of curves whose dual graph has genus $\leq g-i$. Equivalently, $\mathcal{M}_{g, n}^{\lambda_{i}}$ is the locus of curves where the sum of the geometric genera of the components is at least $i$.

Definition 1.3. $\mathcal{M}_{g, n}^{\mathrm{ch}_{2 i-1}} \subseteq \overline{\mathcal{M}}_{g, n}$ is the locus of curves with at least one component of genus at least $i$.

Lemma 1.4. $\mathcal{M}_{g, n}^{\lambda_{g}}=\mathcal{M}_{g, n}^{c t}$ and $\mathcal{M}_{g, n}^{\mathrm{ch}_{2 g-1}}=\mathcal{M}_{g, n}^{r t}$.

Proof. The dual graph of any curve in $\mathcal{M}_{g, n}^{\lambda_{g}}$ is connected of genus 0 and thus is a tree. Any curve in $\mathcal{M}_{g, n}^{\mathrm{ch}_{2 g-1}}$ has at least one (hence exactly one) component of genus $g$; the other components must necessarily form trees of rational curves.

Lemma 1.5. For $i=1,2, \ldots, g$,

(1) the class $\lambda_{i}$ vanishes on $\overline{\mathcal{M}}_{g, n} \backslash \mathcal{M}_{g, n}^{\lambda_{i}}$,

(2) the class $\mathrm{ch}_{2 i-1}$ vanishes on $\overline{\mathcal{M}}_{g, n} \backslash \mathcal{M}_{g, n}^{\mathrm{ch}_{2 i-1}}$.

Proof. The boundary stratum $\delta_{\Gamma}$ is the image of the gluing morphism

$$
\delta_{\Gamma}=\operatorname{im}\left(\iota_{\Gamma}: \prod \overline{\mathcal{M}}_{g_{j}, n_{j}+d_{j}} \rightarrow \overline{\mathcal{M}}_{g, n}\right) .
$$

The Hodge bundle splits when restricted to $\delta_{\Gamma}$ as in [FP1, equations (17) and (18)]:

$$
\iota_{\Gamma}^{*}(\mathbb{E})=\bigoplus \mathbb{E}_{g_{j}, n_{j}+d_{j}} \oplus \mathcal{O}^{n} .
$$

To see (1), use the Whitney formula,

$$
\iota_{\Gamma}^{*}(c(\mathbb{E}))=c\left(\iota_{\Gamma}^{*} \mathbb{E}\right)=\prod\left(1+\lambda_{1, j}+\cdots+\lambda_{g_{j}, j}\right),
$$

where $\lambda_{i, j}$ is the $i$-th Chern class of the Hodge bundle of the $j$-th factor $\overline{\mathcal{M}}_{g_{j}, n_{j}+d_{j}}$. If $\delta_{\Gamma} \in \overline{\mathcal{M}}_{g, n} \backslash \mathcal{M}_{g, n}^{\lambda_{i}}$, then $\sum g_{j}<i$ and the term of degree $i$ in equation (25) vanishes.

To see (2), use the additivity of the Chern character, on $\delta_{\Gamma}$ :

$$
\iota_{\Gamma}^{*}\left(\operatorname{ch}_{2 i-1}(\mathbb{E})\right)=\operatorname{ch}_{2 i-1}\left(\iota_{\Gamma}^{*} \mathbb{E}\right)=\sum_{j} \operatorname{ch}_{2 i-1, j},
$$




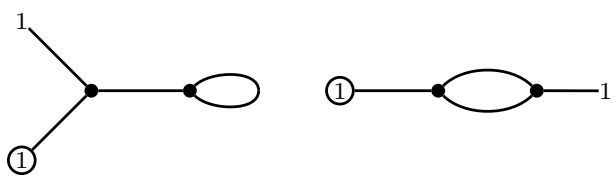

Figure 1. These two dual graphs differ by a Feynman move. Our notation has the genus of a component inside the corresponding vertex, unless the component is rational, in which case it is denoted by a black vertex.

where $\operatorname{ch}_{2 i-1, j}$ is the $(2 i-1)$-th Chern character of the Hodge bundle of the $j$-th factor $\overline{\mathcal{M}}_{g_{j}, n_{j}+d_{j}}$. Since $\delta_{\Gamma} \in \overline{\mathcal{M}}_{g, n} \backslash \mathcal{M}_{g, n}^{\mathrm{ch}_{2 i-1}}$, all $g_{j}<i$ and thus $\mathrm{ch}_{2 i-1}=0$.

We conclude this section by recalling theorem $\star$ by Graber-Vakil (GV2]), which is a key ingredient in the proof of Proposition 1

Theorem $\star$. Any tautological class of degree $k$ on $\mathcal{M}_{g, n}$ vanishes on the open set consisting of strata satisfying

$$
\#\{\text { genus } 0 \text { components }\}<k-g+1 \text {. }
$$

\section{SOCLE}

In this section we prove Proposition 1. The strategy of the proof is natural: Theorem $\star$ forces tautological classes of high degree to be supported on strata with many rational components. On the other hand, curves in $\mathcal{M}_{g, n}^{\lambda_{i}}$ and $\mathcal{M}_{g, n}^{\mathrm{ch}_{2 i-1}}$ satisfy geometric conditions that limit the number of rational components. These constraints imply high degree vanishing and force tautological classes in the socle degree to be supported on exactly one boundary stratum up to rational equivalence.

Definition 2.1. A Feynman move replaces a portion of a graph of type (a) on a dual graph with one of type (b) or (c), as illustrated below:

(a)

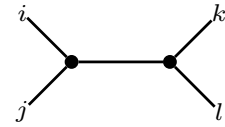

(b)

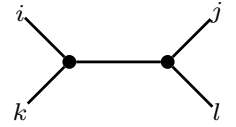

(c)

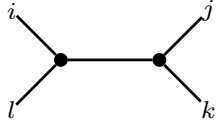

The half-edges $i, j, k$, and $l$ may be glued to other half-edges to form edges (see Figure 1).

Lemma 2.2. If the dual graphs of two boundary strata differ by Feynman moves, then they are rationally equivalent.

Proof. This is immediate by noting that $\overline{\mathcal{M}}_{0,4} \cong \mathbb{P}_{1}$, so its boundary points are rationally equivalent. This equivalence is preserved under gluing morphisms.

Remark 5. It is a standard combinatorial fact that two trivalent graphs with the same number of vertices and edges differ by a finite number of Feynman moves ([W]).

The proof of Proposition 1 now follows from some careful bookkeeping.

Part 1. Theorem $\star$ implies that any class in $R^{3 g-3+n-i+k}\left(\mathcal{M}_{g, n}^{\lambda_{i}}\right)(k \geq 0)$ must be supported on boundary strata containing at least $2 g+n-2-i+k$ rational components. Let $\delta_{\Gamma}$ be any one such boundary stratum. By the definition of $\mathcal{M}_{g, n}^{\lambda_{i}}$, the sum of the genera of the vertices of graph $\Gamma$ is at least $i$. 
Stability implies that the incidence of any rational vertex is at least three, and there are at most $n$ marked half-edges incident to these vertices. Thus there are at least

$$
3(2 g+n-2-i+k)-n=6 g+2 n-6-3 i+3 k
$$

half-edges which must be glued to other half-edges. Since $g_{\Gamma} \leq g-i$, at least

$$
6 g+2 n-6-3 i+3 k-2(3 g+n-2-2 i+k)=i+k
$$

half-edges must be glued to vertices of positive genus. The total genus of the curve represented by $\Gamma$ is $g$, so the only possibility is $k=0$; i.e. all rational vertices are trivalent, and the remaining $i$ half-edges are glued to $i$ vertices of genus 1 and incidence 1. By Remark 5, any two such graphs differ by a finite number of Feynman moves and hence represent rationally equivalent tautological classes. It is immediate to check that such graphs actually live in degree $3 g-3+n-i$.

Part 2. This is similar to the previous proof, except that now we require the graph $\Gamma$ to have at least one vertex of genus $\geq i$. Theorem $\star$ forces any class in $R^{3 g+n-2-2 i+k}\left(\mathcal{M}_{g, n}^{\mathrm{ch}_{2 i-1}}\right)(k \geq 0)$ to be supported on boundary strata with at least $2 g+n-1-2 i+k$ rational components, and stability implies that there are at least three times as many half-edges, only $n$ of which are not glued to some other halfedge. The total genus of the graph must be $g$, where the only consistent possibility is for $k=0$; i.e., all rational vertices are trivalent and there is exactly one vertex of genus $i$ and incidence 1. Again, all such strata represent rationally equivalent classes because any two graphs are equivalent up to a finite number of Feynman moves.

The degree of such strata is $3 g+n-1-3 i$, which means that one must have a class of degree $i-1$ decorating the genus $i$ vertex. Since $R^{i-1}\left(\mathcal{M}_{i, 1}^{\mathrm{rt}}\right) \cong \mathbb{Q}$ (this is the socle statement of Faber's conjecture for $\left.\mathcal{M}_{i, 1}^{\mathrm{rt}}\right)$, there is only one such nonzero class up to scalar multiples.

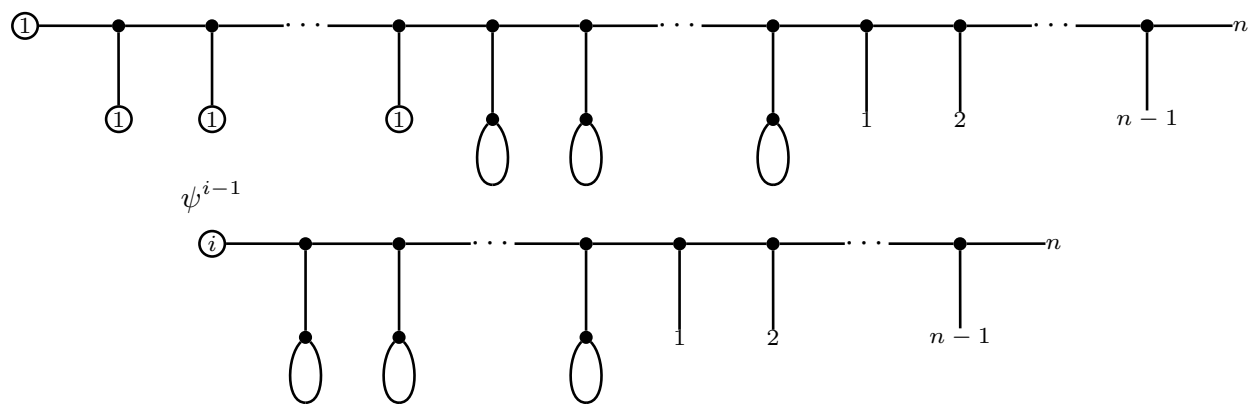

FiguRE 2. Generators in socle degree for $\mathcal{M}_{g, n}^{\lambda_{i}}$ and $\mathcal{M}_{g, n}^{\mathrm{ch}_{i}}$.

Remark 6. It is important to note that $\lambda_{i}$ (resp. $\left.\mathrm{ch}_{2 i-1}\right)$ does not vanish on the unique generator of $R^{t o p}\left(\mathcal{M}_{g, n}^{\lambda_{i}}\right)$ (resp. $R^{t o p}\left(\mathcal{M}_{g, n}^{\mathrm{ch}_{2 i-1}}\right)$ ), and hence it can be used as an evaluation class: multiplication by $\lambda_{i}\left(\right.$ resp. $\left.\mathrm{ch}_{2 i-i}\right)$ gives an isomorphism between the socle and $R^{3 g-3+n}\left(\overline{\mathcal{M}}_{g, n}\right)$. 


\section{Failure of Poincaré duality for $\mathcal{M}_{g, n}^{\lambda_{1}}$}

In this section we construct examples to illustrate the failure of Poincaré duality $\mathcal{M}_{g, n}^{\lambda_{1}}=\mathcal{M}_{g, n}^{\mathrm{ch}_{1}}$ for $g \geq 2$ and $(g, n) \neq(2,0)$. Choose any triple $(a, b, c)$ of integers satisfying $a+b+c=g$, where $a$ is nonnegative and $b$ and $c$ are positive. Choose any subset $S$ of the $n$ points. Let $\Gamma(a, b, c, S) \in R^{g}\left(\overline{\mathcal{M}}_{g, n}\right)$ denote the graph with two vertices connected by $b$ edges: one genus 1 vertex with $a$ self-edges and carrying the points in $S$, and one genus 0 vertex with $c$ self-edges and carrying the points in $S^{c}$ (see Figure 3). Let $\delta(a, b, c, S)$ denote the associated boundary stratum.

When $(a, c, S) \neq\left(c-1, a+1, S^{c}\right)$, the strata $\delta(a, b, c, S)$ and $\delta\left(c-1, b, a+1, S^{c}\right)$ are not rationally equivalent, but their difference lies in the kernel of multiplication by $\lambda_{1}$.

Proof of Proposition 2. Let $\gamma_{1}=\delta(a, b, c, S)$ and $\gamma_{2}=\delta\left(c-1, b, a+1, S^{c}\right)$. Note that if $(a, c, S)=\left(c-1, a+1, S^{c}\right)$, then $\gamma_{1}$ and $\gamma_{2}$ denote the same graph. Therefore assume that this is not the case; we can always do this if $g \geq 2$ and $(g, n) \neq(2,0)$. The fact that $\gamma_{1}-\gamma_{2}$ lies in the kernel of the map $\phi$ from (41) follows from the fact that $\lambda_{1}$ vanishes on $\overline{\mathcal{M}}_{0, k}$ and $\lambda_{1}$ is equivalent to $\frac{1}{24} \delta_{\text {irr }}$ on $\overline{\mathcal{M}}_{1, k}$, for all $k$ for which these spaces are defined. Thus $\lambda_{1} \cdot \gamma_{1}$ and $\lambda_{1} \cdot \gamma_{2}$ are both equal to $\frac{1}{24} \delta_{A}$. Here $A$ is the graph with two genus 0 vertices connected by $b$ edges: one has marked points indexed by $S$ and $a+1$ self-loops, and the other has marked points indexed by $S^{c}$ and $c$ self-loops.

Suppose for a contraction that $\gamma_{1}$ and $\gamma_{2}$ were algebraically equivalent in $\mathcal{M}_{g, n}^{\lambda_{1}}$. The restriction sequence

$$
R^{0}\left(\overline{\mathcal{M}}_{g, n} \backslash \mathcal{M}_{g, n}^{\lambda_{i}}\right) \longrightarrow R^{g}\left(\overline{\mathcal{M}}_{g, n}\right) \longrightarrow R^{g}\left(\mathcal{M}_{g, n}^{\lambda_{i}}\right) \longrightarrow 0
$$

is exact since the first term has degree 0 . Extending $\gamma_{1}$ and $\gamma_{2}$ to boundary strata in $\overline{\mathcal{M}}_{g, n}$,

$$
\gamma_{1}-\gamma_{2} \in R^{0}\left(\overline{\mathcal{M}}_{g, n} \backslash \mathcal{M}_{g, n}^{\lambda_{1}}\right) .
$$

However $R^{0}\left(\overline{\mathcal{M}}_{g, n} \backslash \mathcal{M}_{g, n}^{\lambda_{1}}\right)$ is generated by one element $\delta_{B}$, where $B$ is the graph with a unique vertex of genus $0, g$ self-loops and $n$ half-edges.

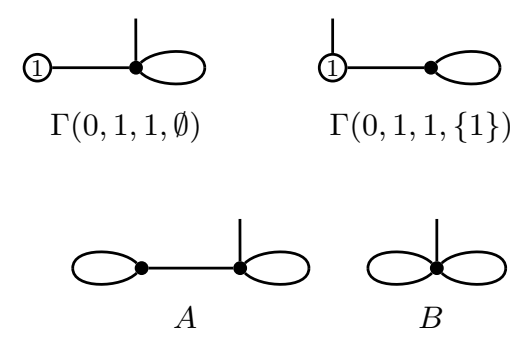

Figure 3 . The graphs $\Gamma(0,1,1, \emptyset)$ and $\Gamma(0,1,1,\{1\})$ for $(g, n)=$ $(2,1)$, and the corresponding graphs $A$ and $B$. 
Set

$$
\begin{array}{ll}
K_{1}:=2 a+b+|S|, & L_{1}:=2 c+b+\left|S^{c}\right|-3, \\
K_{2}:=2 c+b+\left|S^{c}\right|-2, & L_{2}:=2 a+b+|S|-1 .
\end{array}
$$

For $i=1,2$ define the stratum

$$
\gamma_{i}:=\operatorname{im}\left(\iota_{\Gamma_{i}}: \overline{\mathcal{M}}_{1, K_{i}} \times \overline{\mathcal{M}}_{0, L_{i}+3} \rightarrow \overline{\mathcal{M}}_{g, n}\right)
$$

and

$$
\delta_{B}:=\operatorname{im}\left(\iota_{B}: \overline{\mathcal{M}}_{0,2 g+n} \rightarrow \overline{\mathcal{M}}_{g, n}\right) .
$$

Note that $L_{1}$ and $L_{2}$ cannot both be zero for $(g, n) \neq(2,0)$. If they were, then necessarily $a=0, b=1$, and $c=1$ (recall that $a$ is nonnegative while $b$ and $c$ are positive), which implies $(g, n)=(2,0)$.

If $L_{1}=0$, then $L_{2} \neq 0$ and we have the following equations which follow from Lemma 1.1 and the fact that $\kappa_{a}$ restricted to a boundary divisor is the sum of the pull-backs of $\kappa_{a}$ on each factor of the gluing map:

$$
\begin{aligned}
\kappa_{K_{1}} \gamma_{1} & =\frac{1}{2^{g-1}(g-2) !} \frac{1}{24}, & \kappa_{1} \kappa_{L_{2}} \gamma_{1} & =\frac{1}{2^{g-1}(g-2) !} \frac{1}{24}\left(\begin{array}{c}
2 g+n-2 \\
2
\end{array}\right), \\
\kappa_{K_{1}} \gamma_{2} & =0, & \kappa_{1} \kappa_{L_{2}} \gamma_{2} & =\frac{1}{2^{g-1}(g-1) !} \frac{1}{24}, \\
\kappa_{K_{1}} \delta_{B} & =\frac{1}{2^{g} g !}, & \kappa_{1} \kappa_{L_{2}} \delta_{B} & =\frac{1}{2^{g} g !}\left(\left(\begin{array}{c}
2 g+n-1 \\
2
\end{array}\right)-1\right) .
\end{aligned}
$$

These are incompatible with equation (31). If $L_{2}=0$ and $L_{1} \neq 0$, a similar argument holds.

Now consider the final case where both $L_{1}$ and $L_{2}$ are nonzero. The equations

$$
\begin{aligned}
& \kappa_{K_{1}} \kappa_{L_{1}} \gamma_{1}=\frac{1}{24}, \quad \kappa_{K_{1}} \kappa_{L_{1}} \gamma_{2}=0, \quad \kappa_{K_{1}} \kappa_{L_{1}} \delta_{B}=\left(\begin{array}{c}
2 g+n-1 \\
K_{1}+1
\end{array}\right)-1, \\
& \kappa_{2 g-3+n} \gamma_{1}=0, \quad \kappa_{2 g-3+n} \gamma_{2}=0, \quad \kappa_{2 g-3+n} \delta_{B}=\frac{1}{2^{g} g !}
\end{aligned}
$$

show the independence of the strata $\gamma_{1}-\gamma_{2}$ and $\delta_{B}$. Thus $\gamma_{1}$ and $\gamma_{2}$ cannot be albegraically equivalent in $\mathcal{M}_{g, n}^{\lambda_{1}}$.

Corollary 5. The dimension of the kernel of the map

$$
\phi: R^{g}\left(\mathcal{M}_{g, n}^{\lambda_{1}}\right) \rightarrow \operatorname{hom}\left(R^{2 g-4+n}\left(\mathcal{M}_{g, n}^{\lambda_{1}}\right), R^{3 g-4+n}\left(\mathcal{M}_{g, n}^{\lambda_{1}}\right)\right)
$$

goes to infinity as $g$ or $n$ go to infinity.

Proof. We exhibit a set of roughly $g+n / 2$ linearly independent classes in ker $\phi$. Let $\bar{n}$ denote the set $\{1, \ldots, n\}$. Set

$$
\gamma_{i}=\delta(0,1, g-1, \overline{2 i})-\delta(g-2,1,1, \bar{n} \backslash \overline{2 i})
$$

for $i=1, \ldots,\lfloor n / 2\rfloor$, and

$$
\eta_{j}=\delta(j, 1, g-j-1, \bar{n})-\delta(g-j-2,1, j+1, \emptyset)
$$




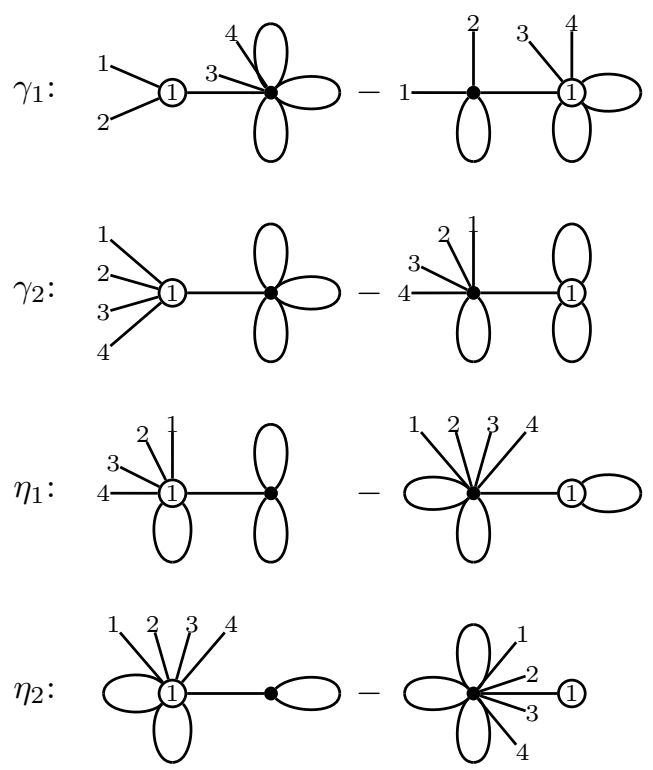

Figure 4 . The classes $\gamma_{1}, \gamma_{2}, \eta_{1}$, and $\eta_{2}$ for $(g, n)=(4,4)$

for $j=1, \ldots, g-2$. Since $\lambda_{1} \gamma_{i}=\lambda_{1} \eta_{j}=0$, the classes lie in the kernel of $\phi$. The equations

$$
\begin{aligned}
& \psi_{1}^{2 i+1} \kappa_{2 g+n-4-2 i} \gamma_{j}=A_{i} \delta_{i j}, \\
& \psi_{1}^{2 i+1} \kappa_{2 g+n-4-2 i} \eta_{j}=0, \\
& \psi_{1}^{n+2 i+1} \kappa_{2 g-4-2 i} \gamma_{j}=0, \\
& \psi_{1}^{n+2 i+1} \kappa_{2 g-4-2 i} \eta_{j}=B_{i} \delta_{i j}
\end{aligned}
$$

(here $\delta_{i j}$ is Kronecker's delta and not a boundary stratum, and $A_{i}, B_{i}$ are nonzero real numbers) show that these classes are independent. Modulo $R^{0}\left(\overline{\mathcal{M}}_{g, n} \backslash \mathcal{M}_{g, n}^{\lambda_{1}}\right)$, which is one-dimensional, roughly $g+\lfloor n / 2\rfloor-1$ of them must remain independent.

\section{Failure of Poincaré duality for $\mathcal{M}_{g, n}^{\lambda_{i}}$}

We first outline the strategy of the proof for Proposition 3 Fix $g$ and $i$ such that $2 \leq i \leq g-1$. A generalization of the construction in $\$ 3$ produces a set $S_{m} \subseteq R^{g}\left(\overline{\mathcal{M}}_{g,(i+1)+m}\right)$ in the annihilator of $\lambda_{i}$. The first problem in showing that at least one such class is nonzero in $R^{g}\left(\mathcal{M}_{g,(i+1)+m}^{\lambda_{i}}\right)$ is that the kernel of the restriction sequence (6) is not known to be tautological. This is a difficult question that we cannot tackle at present. If the kernel is in fact tautological, the dimension of $R^{i-1}\left(\overline{\mathcal{M}}_{g, n} \backslash \mathcal{M}_{g, n}^{\lambda_{i}}\right)$ grows quickly as $g$ or $n$ increases. For a fixed $g$ we bound the order of growth by $i^{n}$. By proving that $S_{m}$ spans a linear subspace of dimension $(i+1)^{m}$, we conclude that eventually some classes in $S_{m}$ cannot lie in the image of $R^{i-1}\left(\overline{\mathcal{M}}_{g, n} \backslash \mathcal{M}_{g, n}^{\lambda_{i}}\right)$ and hence will be nonzero in $R^{g}\left(\mathcal{M}_{g,(i+1)+m}^{\lambda_{i}}\right)$. 


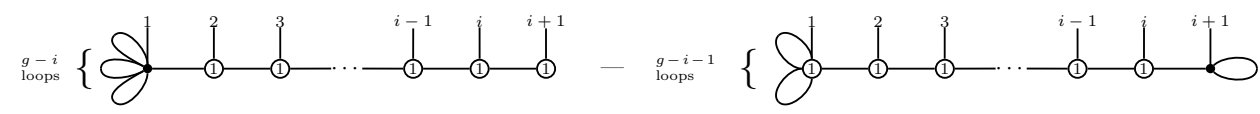

Figure 5. The class $\sigma \in R^{g}\left(\mathcal{M}_{g, i+1}^{\lambda_{i}}\right)$. There are $g-i$ loops on each stratum. On the left hand side all loops are incident to the rational vertex; on the right hand side $g-i-1$ are incident to a genus one vertex and one loop connects the rational vertex to itself.

Proof of Proposition 3. Let $\sigma \in R^{g}\left(\overline{\mathcal{M}}_{g, i+1}\right)$ be the difference of boundary classes illustrated in Figure 5. Intersecting either of the two strata with $\lambda_{i}$ results in $1 / 24^{i}$ times the class of the graph where all genus one vertices are replaced with loops, and thus $\sigma \cdot \lambda_{i}=0$. We set $S_{0}=\{\sigma\}$.

For $m>0$, and $\mathbf{a}=\left(a_{1}, \ldots, a_{m}\right)$ an $m$-tuple of numbers between 1 and $i+1$, let $\sigma_{\mathbf{a}} \in R^{g}\left(\overline{\mathcal{M}}_{g,(i+1)+m}\right)$ be the class obtained by decorating both graphs of $\sigma$ with the $j$-th mark on the $a_{j}$-th vertex for $j=1, \ldots, m$. The set $S_{m}$ of all possible such classes in $\mathcal{M}_{g,(i+1)+m}^{\lambda_{i}}$ has cardinality $(i+1)^{m}$.

We construct inductively a set $T^{m}$ of classes of $R^{2 g-3+(i+1)+m}\left(\overline{\mathcal{M}}_{g,(i+1)+m}\right)$ which is dual to $S_{m}$. Our base case is $m=0$, where the vector $\tau$ can be chosen to be a scalar multiple of an appropriate product of $\psi$ classes (for example, $\tau$ can be defined by normalizing $\psi_{1}^{2(g-i-1)} \psi_{i+1}^{2} \prod_{j=2}^{i} \psi_{j}^{3}$ ).

We describe the first inductive step explicitly to illustrate the general step. In this case, $S_{1}=\left\{\sigma_{k}\right\}_{k=1, \ldots, i+1}$, where $\sigma_{k}$ is obtained by decorating $\sigma$ with the $(i+2)$ th point on the $k$-th vertex. The dual set $T^{1}=\left\{\tau^{k}\right\}_{k=1, \ldots, i+1}$ is constructed as follows: $\tau^{k}$ is the pull-back via the universal family of the class $\tau$, intersected with the boundary divisor where the $k$-th and the $((i+1)+1)$-th point are on a genus 0 component.

In general consider the universal family

$$
\pi: \overline{\mathcal{M}}_{g,(i+1)+m+1} \rightarrow \overline{\mathcal{M}}_{g,(i+1)+m},
$$

and note that

$$
\pi^{*} \sigma_{\mathbf{a}}=\sum_{k=1}^{i+1} \sigma_{(\mathbf{a}, k)},
$$

where $(\mathbf{a}, k)$ is the sequence with $k$ appended to the end of $\mathbf{a}$. For $1 \leq j \leq(i+1)$ let $D_{j,(i+1)+m+1}$ denote the divisor image of the $j$-th section in $\overline{\mathcal{M}}_{g,(i+1)+m+1}$ (i.e. the boundary divisor where the $j$-th and the last mark are sitting on a genus 0 component). Then, for any $m$-tuple a,

$$
D_{j,(i+1)+m+1} \cdot \sigma_{(\mathbf{a}, k)}=0
$$

if $j \neq k$. By the projection formula and equations (49) and (50),

$$
D_{j,(i+1)+m+1} \pi^{*}\left(\tau^{\mathbf{b}}\right) \sigma_{(\mathbf{a}, k)}
$$


equals the class of a point if $j=k$ and $\mathbf{a}=\mathbf{b}$ and vanishes otherwise. Therefore the set

$$
T^{m+1}:=\left\{\pi^{*} \tau^{b_{1}, \ldots, b_{m}} D_{b_{m+1},(i+1)+m+1}\right\}_{\mathbf{b}}
$$

gives a dual basis to $S_{m+1}$.

The growth of $\operatorname{dim} R^{i-1}\left(\overline{\mathcal{M}}_{g, n} \backslash \mathcal{M}_{g, n}^{\lambda_{i}}\right)$ with respect to $n$ is at most $O\left(i^{n}\right)$. To see this, note that the decorated dual graph of any class in $R^{i-1}\left(\overline{\mathcal{M}}_{g, n} \backslash \mathcal{M}_{g, n}^{\lambda_{i}}\right)$ has at most $i$ vertices, and $e$ edges, where $g-i+1 \leq e \leq g$. The total number of possibly unstable graphs without marked points satisfying these conditions is independent of $n$.

Graphs with exactly $i$ vertices have $g$ edges, and hence classes supported on these strata are pure boundary. For a given graph there are $i^{n}$ possible ways of distributing the marked points on the vertices.

For graphs with strictly less than $i$ vertices, there are at most $(i-1)^{n}$ ways to distribute the marks on the vertices. Each vertex can be decorated with a monomial in $\psi$ and $\kappa$ classes of degree $\leq i-1$ (GP, Proposition 11]). The number of ways to choose $\kappa$ classes to decorate the vertices is independent of $n$. The number of monomials in $\psi$ classes of degree $\leq i-1$ grows polynomially in $n$, yielding an order of $O\left((i-1)^{n} n^{k}\right)<O\left(i^{n}\right)$ for the number of classes supported on strata with less than $i$ vertices.

Altogether, we have obtained that the dimension of $R^{i-1}\left(\overline{\mathcal{M}}_{g, n} \backslash \mathcal{M}_{g, n}^{\lambda_{i}}\right)$ grows at most as $O\left(i^{n}\right)$. Thus $\operatorname{dim} R^{g}\left(\mathcal{M}_{g, n}^{\lambda_{i}}\right)>\operatorname{dim} R^{i-1}\left(\overline{\mathcal{M}}_{g, n} \backslash \mathcal{M}_{g, n}^{\lambda_{i}}\right)$ for large $n$, which implies that some classes in the annihilator of $\lambda_{i}$ are nonzero in $R^{g}\left(\mathcal{M}_{g, n}^{\lambda_{i}}\right)$.

All nontrivial classes in the annihilators of $\lambda_{i}$ that we construct are in codimension $g$ or higher. This leaves us with a natural question:

Question. Is the map

$$
\phi: R^{j}\left(\mathcal{M}_{g, n}^{\lambda_{i}}\right) \rightarrow \operatorname{hom}\left(R^{3 g-3+n-i-j}\left(\mathcal{M}_{g, n}^{\lambda_{i}}\right), R^{3 g-3+n-i}\left(\mathcal{M}_{g, n}^{\lambda_{i}}\right)\right)
$$

injective for $j \leq g-1$ ?

\section{REFERENCES}

[AC] Enrico Arbarello and Maurizio Cornalba, Combinatorial and algebro-geometric cohomology classes on the moduli spaces of curves, J. Algebraic Geom. 5 (1996), no. 4, 705-749. MR:1486986 (99c:14033)

[Fa] Carel Faber, A conjectural description of the tautological ring of the moduli space of curves, Moduli of curves and abelian varieties, Aspects Math., E33, Vieweg, Braunschweig, 1999, pp. 109-129. MR.1722541 (2000j:14044)

[FP1] Carel Faber and Rahul Pandharipande, Hodge integrals and Gromov-Witten theory, Invent. Math. 139 (2000), no. 1, 173-199. MR.1728879 (2000m:14057)

[FP2] _ Logarithmic series and Hodge integrals in the tautological ring, Michigan Math. J. 48 (2000), 215-252. With an appendix by Don Zagier; dedicated to William Fulton on the occasion of his 60th birthday. MR1786488 (2002e:14041)

[FP3] - Relative maps and tautological classes, J. Eur. Math. Soc. (JEMS) 7 (2005), no. 1, 13-49. MR2120989 (2005m:14046)

[Fu] William Fulton, Intersection theory, 2nd ed., Ergebnisse der Mathematik und ihrer Grenzgebiete. 3. Folge. A Series of Modern Surveys in Mathematics [Results in Mathematics and Related Areas. 3rd Series. A Series of Modern Surveys in Mathematics], vol. 2, Springer-Verlag, Berlin, 1998. MR,1644323 (99d:14003)

[GP] Tom Graber and Rahul Pandharipande, Constructions of nontautological classes on moduli spaces of curves, Michigan Math. J. 51 (2003), no. 1, 93-109. MR1960923 $(2004 \mathrm{e}: 14043)$ 
[GV1] Tom Graber and Ravi Vakil, On the tautological ring of $\overline{\mathcal{M}}_{g, n}$, Turkish J. Math. 25 (2001), no. 1, 237-243. MR.1829089 (2002b:14034)

[GV2] _ Relative virtual localization and vanishing of tautological classes on moduli spaces of curves, Duke Math. J. 130 (2005), no. 1, 1-37. MR2176546 (2006j:14035)

[HM] Joe Harris and Ian Morrison, Moduli of curves, Graduate Texts in Mathematics, vol. 187, Springer-Verlag, New York, 1998. MR1631825 (99g:14031)

[HKK $\left.{ }^{+}\right]$Kentaro Hori, Sheldon Katz, Albrecht Klemm, Rahul Pandharipande, Richard Thomas, Cumrun Vafa, Ravi Vakil, and Eric Zaslow, Mirror symmetry, Clay Mathematics Monographs, vol. 1, American Mathematical Society, Providence, RI; Clay Math. Institute, Cambridge, MA, 2003. With a preface by Vafa. MR2003030 (2004g:14042)

[Ke] Sean Keel, Intersection theory of moduli space of stable $n$-pointed curves of genus zero, Trans. Amer. Math. Soc. 330 (1992), no. 2, 545-574. MR.1034665 (92f:14003)

[Ko] Joachim Kock, Notes on psi classes, available at http://www.mat.uab.es/ kock/ GW.html.

[L] Eduard Looijenga, On the tautological ring of $\mathcal{M}_{g}$, Invent. Math. 121 (1995), no. 2, 411-419. MR1346214 (96g:14021)

[M] David Mumford, Towards an enumerative geometry of the moduli space of curves, Arithmetic and geometry, Vol. II, Progr. Math., vol. 36, Birkhäuser Boston, Boston, MA, 1983, pp. 271-328. MR717614 (85j:14046)

[W] J. H. C. Whitehead, On $C^{1}$-complexes, Ann. of Math. (2) 41 (1940), 809-824. MR0002545 (2,73d)

[Y] Stephanie Yang, Intersection numbers on $\overline{\mathcal{M}}_{g, n}$. Preprint. Available at http://www. stephanieyang.com/Papers.html.

Department of Mathematics, Colorado State University, Weber Building, ForT Collins, Colorado 80523-1874

E-mail address: renzo@math.colostate.edu

Institutionen för Matematik, Kungliga Tekniska Högskolan, 10044 Stockholm, SWEDEN

E-mail address: stpyang@math.kth.se 